\title{
Assessment of rice leaf chlorophyll content using visible bands at different growth stages at both the leaf and canopy scale
}

\begin{abstract}
Nitrogen is an important variable for paddy farming management. The objectives of this study were to develop and test a new method to determine the status of nitrogen and chlorophyll content in rice leaf by analysing and considering all visible bands derived from images captured using a conventional digital camera. The images from the 6-pannel leaf colour chart were acquired using Basler Scout scA640-70fc under light-emitting diode lighting, in which principal component analysis was used to retain the lower order principal component to develop a new index. Digital photographs of the upper most collared leaf of rice (Oriza sativa L.), grown over a range of soils with different nitrogen treatments, were processed into 11 indices and IPCA through six growth stages. Also a conventional digital camera mounted to an unmanned aerial vehicle was used to acquire images over the rice canopy for the purpose of verification. The result indicated that the conventional digital camera at the both leaf $(\mathrm{r}=-0.81)$ and the canopy $(\mathrm{r}=0.78)$ scale could be used as a sensor to determine the status of chlorophyll content in rice plants through different growth stages. This indicates that conventional low-cost digital cameras can be used for determining chlorophyll content and consequently for monitoring nitrogen content of the growing rice plant, thus offering a potentially inexpensive, fast, accurate and suitable tool for rice growers. Additionally, results confirmed that a low cost LARS system would be well suited for high spatial and temporal resolution images and data analysis for proper assessment of key nutrients in rice farming in a fast, inexpensive and non-destructive way.
\end{abstract}

Keyword: Conventional digital camera; Image analysis; Low altitude remote sensing; Nitrogen; Principal component analysis; Rice 